The prospect of a perfect ending: Loss aversion and the round-number bias

\begin{abstract}
Studies across a range of domains have shown that individuals tend to focus on round numbers as cognitive reference points; a so-called left-digit effect. We explain this effect by combining analog numerical heuristics with prospect theory in order to develop an analog value function that predicts the key characteristics of the left-digit effect. Most importantly, this value function predicts an unreported phenomenon, namely; that the left-digit effect will be more pronounced in situations involving losses (cf. gains). We confirm this prediction in both a laboratory experiment regarding hypothetical investments and analysis of buy-sell imbalances in over 15 million trades by investors in a financial market. We conclude that our analog value function is a promising explanation for the left-digit effect. Furthermore, we suggest that interventions aimed at reducing costly buy-sell imbalances in financial markets should focus on the decisions made by investors when they are facing loss.
\end{abstract}

Keywords: Analog Value Function; Round-Number Bias; Left-digit Effect; Heuristics 


\section{The prospect of a perfect ending: Loss aversion and the round-number bias}

\section{Introduction}

Research has revealed that small changes in prices can significantly influence individuals' perceptions of an item's cost. The left-digit effect (LDE) or "pricing in the nines" (Basu, 1997; Stiving, 2000) occurs when two prices that differ by 1 cent (e.g., $\$ 2.99$ vs. $\$ 3.00$ ) are encoded differently in the perceiver's mind due to differences in their left digits. This has clear applications in the pricing of commercial goods and services (Manning \& Sprott, 2009; Stiving, 2000; Thomas \& Morwitz, 2005). However, recent studies suggest that the LDE could play a role in a broad range of decision-making domains (e.g., in financial markets, see Bhattacharya, Holden, \& Jacobsen, 2012). In light of the importance of this phenomenon, we develop a theory to explain the observed LDE. In particular, we combine dual-process theories of numerical processing during the encoding of price information (Gordon, 2004; Lemer, Dehance, Spelke, \& Cohen, 2003) and prospect theory's value function (Kahneman \& Tversky, 1979; Tversky \& Kahneman, 1992) to develop a new analog value function.

The analog value function is based on two ideas: (1) individuals may use changes in leftdigits to inform their judgment of changes in value; and (2) changes in left-digits will bias choices more readily in situations involving losses than in situations involving gains. Under the dual-process paradigm, the use of simple left-digit heuristics implies a reliance on intuitive type 1 processing rather than the more deliberative type 2 processing associated with calculating the precise change in value (Evans \& Stanovich, 2013a, Hogarth, 2001; Kahneman \& Frederick, 2002; Payne, Bettman, \& Johnson, 1993; Sloman, 1996; Stanovich \& West, 2000). However, our theory is not based on the premise that there must be a change in reasoning strategy from more normative in the context of gains to heuristic in the context of losses. Rather, we consider prospect theory and propose that the salience of left-digit changes may be exaggerated by loss aversion. The higher salience of left-digits changes associated with losses is expected to lead to a 
greater biasing effect in the judgment of changes in value compared to left-digit changes associated with gains.

Figure 1 illustrates the general structure of our model. Initially, the perceiver processes stimulus information and signals related to the changes in left digits are combined with signals related to changes in value (e.g. percentage change). The degree to which the changes in left digits bias the perceived change in value is determined by how much that signal is weighted in a given choice context (modulated by parameter $k$ in Figure 1). This combined signal is then processed according to prospect theory's value function (shown as $v$ in Figure 1), which results in our proposed analog value function. The final choice probabilities are some function of the comparison of the analog value functions produced by each of the choice alternatives.

[Figure 1 approx here.]

We developed hypotheses derived from predictions based on the analog value function. These hypotheses were then tested by analyzing data drawn from a laboratory study in which participants evaluated the performance of investments that gained in value and those that decreased in value. In accordance with our hypotheses, changes in left digits had a greater biasing effect on participants' judgments of investment performance for losing (vs. winning) investments. In particular, when judging the change in value of investments that decreased in value, if the change in left-digits did not coincide with the true change in value participants were more likely to make errors when selecting the best performing asset. However, when the change in left digits did coincide with the best performing asset, error rates were lower - albeit clearly for the wrong reasons. This effect was only marginally significant for gains, suggesting a weaker LDE for gains, as predicted by our model.

Recent studies have discovered buy-sell imbalances on round numbers in real-world stock transactions (see, Bhattacharya et al., 2012; Kuo, Lin, \& Zhao, 2014). These imbalances suggest that investors in financial markets have a tendency to over-buy or over-sell at specific price points that end in round numbers. The findings of these studies indicated the use of 
heuristics by investors, whereby they focus on the change in left digits rather than on the more mentally costly strategy of calculating accurate changes in value (e.g., percentage returns). Arbitrary characteristics of prices, such as whether the prices are round numbers, are unrelated to the fundamental value of the security. Consequently, a preference for round numbers could be detrimental to investors' profitability. Indeed, Bhattacharya et al. (2012) estimated that such irrational behavior could lead to an aggregated wealth transfer of $\$ 813$ million a year in the New York Stock exchange alone. Critically, however, their theory did not account for the role that loss aversion could play in this effect.

To determine whether decision makers in real world settings also tend to be more biased by changes in left digits in the context of a loss, we examined individual financial spreadtrading account data. The transactions were separated into trades relating to investments that gained in value and trades that decreased in value and then examined the degree of LDE in the buy-sell imbalances for these trades. Consistent with the predictions of our analog value function we observed greater buy-sell imbalances on round numbers for investments that decreased in value, confirming that loss-induced round-number biases have an impact on realworld decisions.

The potential costs of the LDE, as revealed by Bhattacharya et al. (2012), demonstrate its importance. From a theoretical perspective, our findings offer an important modification to prospect theory, suggesting that the analog value function could play an important role in a broad range of everyday decisions. This analog value function is valuable in providing a means of measuring the extent that individuals may suffer from round number bias and when they might be most susceptible to its effects (i.e. when facing losses). Hence, this could enable the targeting of psychological interventions (e.g., via decision-support systems) at appropriate individuals and situations, in order to reduce the costly round-number bias in financial markets; potentially fostering improvements in investor performance and market efficiency. 
Our paper proceeds as follows. We first describe the conceptual background and theories related to the LDE and we show how analog numerical processing may affect investor behavior. We then develop an analog value function to account for the LDE and outline why we expect loss aversion to exacerbate the LDE. Next we develop hypotheses based on the analog value function. We then describe the laboratory experiments and the real-world trading data that we employ to test these hypotheses. In this section, we also present our results. Next we discuss the results and we draw conclusions regarding the theoretical and practical implications of our theory and findings.

\section{Literature Review}

\section{The Left-digit-effect}

Laboratory studies have revealed that prices ending in nine (e.g., \$2.99, \$399) are perceived to be disproportionately smaller than prices one unit (cent, dollar, etc.) higher, provided the additional unit causes the left digit to increase (e.g., \$3.00, \$400; Manning \& Sprott, 2009; Thomas \& Morwitz, 2005). This appears to be because individuals tend to evaluate numbers sequentially, beginning with the left-most digits first (Hinrichs, Yurko, \& Hu, 1981; Poltrock \& Schwartz, 1984).

This LDE appears to have far-reaching implications for a range of different situations. Company revenues increase when goods are priced to capitalize on the LDE, suggesting that, even though consumers are probably aware of this pricing trick, the LDE does disproportionately influence behavior (Monroe, 2003; Stiving \& Winer, 1997). Recently, researchers revealed LDEs in the used car market, whereby changes in the left-most digit of the numbers of miles on the clock resulted in sudden drops in price (Busse, Lacetera, Pope, SilvaRisso, \& Syndor, 2013; Lacetera, Pope, \& Syndor, 2012).

The LDE has also been observed in financial markets. Clustering of prices has been found in limit order quotes ${ }^{1}$ (Ahn, Cai, \& Cheung, 2005; Chiao \& Wang, 2009, Niederhoffer, 1965) as well as in the actual execution price of orders (Harris, 1991; Niederhoffer, 1966). 
Recent studies have shown that the frequency of trades decreases monotonically when the asset price ends in, successively, an integer-dollar, half-dollar, quarter, dime, nickel and penny (Ikenberry \& Weston, 2007), due to a preference for round numbers. Following these observations, Bhattacharya et al. (2012) undertook a detailed analysis of buying and selling behavior to determine if buy (sell) trades cluster at different price points. They observed significant over-buying (-selling) when the price fell (rose) to reach a round number². Bhattacharya et al. (2012) cite a left-digit and threshold trigger effect as an explanation for the buy-sell imbalances on round numbers. In particular, they argue that individuals tend to use quick approximations of changes in values, focusing on the left-most digits (analog heuristics), because to calculate the true changes is more mentally costly. Consequently, when traders set their price thresholds for orders, these orders tend to cluster on round numbers. Given that many investors are likely to share the same preference for round numbers, we can expect a flurry of market orders at certain round number price-points. Kuo et al. (2014) argued that individuals who are more cognitively constrained are most likely to be affected by round number bias and demonstrated that such cognitive shortcuts are associated with lower returns across futures and options markets.

\section{Analog Processing Explanation for LDE}

The use of heuristics to form quick approximations of changes in values (Bhattacharya et al., 2012; Manning \& Sprott, 2009; Thomas \& Morwitz, 2005), aligns strongly with analog processing theory. This theory proposes that humans and animals can process numerical magnitudes using an analog internal scale that is not reliant on words or symbols (Thomas \& Morwitz, 2005). For example, studies have revealed that monkeys can learn to order visual image stimuli based upon the number of discriminable visual elements they contain (Brannon \& Terrance, 1998; Hauser, Carey, \& Hauser, 2000). Equally, humans can process numerical information without possessing words or symbols for numbers. For example, members of 
Amazonian tribes who only have words for "one", "two" and "many", appear able to distinguish larger magnitudes via an analog estimation process (Gordon, 2004; Pica, Lemer, Izard, \& Dehaene, 2004).

Researchers have theorized that humans possess two distinct and dissociated quantitative processing systems (Gordon, 2004; Lemer et al., 2003). The lower level system is a primitive approximating system (analog system) that enables individuals to differentiate and order quantities according to magnitude. For example, even before the acquisition of words for numbers, infants can exhibit approximate number discrimination and comparison abilities (Brannon, 2002; Wynn, Bloom, \& Chiang, 2002). Unlike animals, humans also possess a higher level of numeracy, enabling them to represent, learn, and manipulate specific numbers using words and symbols. This enables the complex calculations required for higher-level mathematics (Lemer et al., 2003).

The LDE is theorized to occur when two numbers are compared using the analog representation of their magnitudes, allowing rapid and automatic ordering of the numbers. However, it appears that this system operates in left-to-right digit order (Hinrichs, Yurko, \& Hu, 1981; Poltrock \& Schwartz, 1984; Thomas \& Morwitz, 2005) causing a biasing effect (akin to Tversky and Kahneman's (1974) anchoring and adjustment bias) towards the leftmost digits. By anchoring on the 2 in $\$ 2.99$ and not sufficiently adjusting for the .99 cents, $\$ 2.99$ is encoded as being significantly less than $\$ 3.00$ in the analog scale.

The analog processing explanation is a promising general description of the kind of cognitive processes that might cause round-number bias. However, the literature contains no formal descriptions of analog numerical heuristics. Consequently, it is difficult to envisage how such a heuristic may interact with decision theories in which the perception of value plays a key role. For example, prospect theory (Kahneman \& Tversky, 1979; Tversky \& Kahneman, 1992) relies heavily on the principle that individuals' make choices based on the change in value from a reference point. Reference points play an important role in some surprising areas. For 
example, it has been found that the finishing times of marathon runners bunch around certain reference point times (Allen, Dechow, Pope, \& Wu, 2015; Markle, Wu, White, \& Sacket, 2015). Since changes in value from a reference point could depend on the use of an analog heuristic, understanding how such a heuristic could interact with prospect theory's other components is important. Indeed, as we explain in the next section, it appears that the manner in which prospect theory accounts for loss aversion has some important implications for the impact of analog heuristics on choices.

\section{Dual-Process Theory and the Rule Selection Problem}

Comparisons of analog heuristic processing and higher level numerical processing have clear parallels with comparisons of type 1 (system 1) versus type 2 (system 2) processing in dual-process theory. The default-interventionist view proposes that information from type 1 reasoning (e.g., left-digit heuristic signals) will tend to occur automatically on first exposure to the stimulus. Subsequently, output from type 2 reasoning (i.e., percentage changes in value) may further contribute to preferences (Evans \& Stanovich, 2013a; Fraser-Mackenzie \& Dror, 2011). However, the resolution process of these different and sometimes opposing information sources appears to be complex and there are different views in the literature regarding this process (see the debate between Evans and Stanovich (2013b) vs. Kruglanski (2013) and Keren (2013)). A recent proposal is a three-stage dual-process model (Pennycook, Fugelang, \& Koehler, 2015) which proposes that type 2 processing is only triggered when conflict arises from the range of initial type 1 processing outputs. However, Kruglanski and Gigerenzer (2011) question whether a distinction should even be made between type 1 and type 2 processing. Consequently, they make a case for a unimodal theory in which intuitive and deliberative judgments are both based on rules that may be heuristic or optimizing in nature. The accuracy, therefore, of both deliberate and intuitive judgments depends on the ecological rationality of the rule given the class of problem. In our study, we do not attempt to model an individual's 
selection between the signals generated by the left-digit heuristic and those generated from more deliberative calculations of the change in value. We also do not attempt to capture the possible differences in the temporal ordering of the left-digit and percentage change signals in our model (i.e. that left-digit heuristic signals are probably produced before the percentage change signals). Rather, we simply aim to model the degree to which changes in left digits tend to bias choices at the aggregated population level and whether this bias is indeed greater for judgments involving loss rather than profit. Prospect theory provides a suitable framework to achieve this aim.

\section{Developing an Analog Editing Heuristic for Prospect Theory}

According to prospect theory (Kahneman \& Tversky, 1979), individuals may employ heuristics to simplify choice problems during an 'editing' phase. Kahneman and Tversky (1979) originally suggested that this might involve individuals rounding values to the nearest number (e.g., rounding both 1.99 and 2.01 to 2.00 ). However, such rounding would negate any LDE, as there would no longer be any difference between 1.99 and 2.01, since both would be rounded to 2.00. Clearly, the findings discussed above indicate that this is not the case. Rather, we suggest that individuals may sometimes employ analog processing during this editing phase using some form of an analog heuristic. We outline the process we envisage in this section.

In prospect theory, the input, $x$, is the change from a reference point, $R$, given the current price, $P$. This is an 'unedited' input value.

$$
x=\delta(P, R)=P-R
$$

However, consider the following simple analog heuristic function $(h)$ that provides a different, now 'edited', input:

$$
x=h(P, R, k)=(1-k)(P-R)+k(\mathrm{H}(P, R)),
$$

where $\mathrm{H}$ is some function capturing analog processing and $k$ is a weighting parameter between 0 and 1 determining the degree to which the analog heuristic influences an individual's perception of change in value relative to the actual change in value. If $k=0$ then there is no 
analog heuristic applied and the perception of change in value is identical to Eq. 1. If $k=1$ then the function is equivalent to only calculating the difference in terms of the change in left digit (i.e., a step function).

We include $k$ as a means of varying the extent of the analog heuristic because recent studies reveal that the use of left-digit heuristics can increase or decrease under different conditions. In particular, Kuo et al. (2014) argue that the LDE should be greater amongst individuals that are more cognitively constrained. Indeed, they demonstrated that less experienced and individual (cf. institutional) investors displayed greater round number bias. Consequently, we expect that some individuals have a greater propensity to be influenced by the left digit (for them, $k$ would be higher). Equally, the impact of the left-digit on choices may vary within individuals in different situations; as such, $k$ may vary between situations for the same individual. For example, we would expect factors such as time pressure, stress and cognitive load to increase the impact of the LDE, just as these factors increase the use of other heuristics over more normative reasoning approaches (see Fraser-Mackenzie \& Dror, 2011; Hogarth, 2001; Kahneman \& Frederick, 2002; Payne, Bettman, \& Johnson, 1993; Sloman, 1996; Stanovich \& West, 2000).

Consider the case of evaluating values from 1.00 to 9.99. Individuals might perceive the integer to the left of the decimal point as the left digit and the remaining digits as the right digits. Hence, a simple way to introduce analog processing into Eq. 2 for this scenario could be to use the difference between the floor (i.e. largest previous integer) of the payoff $(\mathrm{P})$ and the floor of the reference point $(\mathrm{R})^{3}$;

$$
\mathrm{H}(P, R)=\lfloor P\rfloor-\lfloor R\rfloor .
$$

As we shall explain, this simple addition of an analog heuristic into prospect theory leads to some interesting predictions when we apply the value function - particularly with respect to loss aversion.

\section{Loss-Aversion and the Analog Heuristic}


Loss aversion is a cornerstone of behavioral finance whereby individuals perceive losses as more serious than equivalent gains (Kahneman \& Tversky, 1979, Tversky \& Kahneman, 1992). However, to the best of our knowledge, no studies have examined the extent to which loss aversion and, in particular, prospect theory's value function, play a role in LDEs.

If we employed our analog heuristic during the editing phase, then two features would become evident once we applied prospect theory's value function during the subsequent 'evaluation' stage. First, we would observe sudden 'jumps' or 'cliffs' in subjective utility when the left digit changes. Second, due to the impact of the value function, these sudden changes in subjective utility are larger for losses than gains. This arises because the value function, $v$, is concave above a reference point $\left(v^{\prime \prime}(x)<0\right.$, for $\left.x>0\right)$ for gains and convex below $\left(v^{\prime \prime}(x)<0\right.$, for $x$ $<0$ ) for losses. As a result, losses are more strongly valued than equivalent gains. An example from Kahneman and Tversky's (1991) value function could be as follows, where $\alpha$ and $\beta$ are parameters to create different degrees of concavity/convexity functions for gains and losses and $\lambda$ is the loss aversion parameter:

$$
v(x)=\left\{\begin{array}{cc}
x^{a} & x \geq 0 \\
-\lambda(-x)^{\beta} & x<0
\end{array}\right.
$$

We illustrate how a typical value function and the analog heuristic behave for changes from different reference points in Figure 2. This figure demonstrates that for losses/gains there are 'cliff edges'/'jumps' in subjective value on round numbers but the size of these jumps are greater in the domain of losses. Interestingly, the size of the steps are greatest around $x=0$ and diminish the further from the reference point the change in the left-digit occurs. This is due to decreasing marginal utility captured by the value function in gains and loss. In other words, the step-size resulting from the LDE decreases as the difference between the values being compared increases. This is a result of the concavity/convexity of prospect theory's value function, which is steepest around the reference point.

[Figure 2 approx. here] 
The function illustrated in Figure 2 can explain why Bhattacharya et al. (2012) observed increased buying-selling imbalances on round numbers. If investors are losing money on an asset and the price of that asset falls to a round number, they are now 'atop a cliff edge'. Consequently, they may decide to sell their asset to avoid the potential large drop in subjective expected value if the price falls below the round number, changing the left-digit (we provide examples of the effect of analog-based editing on the choices of an investor in Appendix A). However, if an investment has gained in value but the investors are at the 'bottom of a cliff' (i.e. a price ends in .99), they may prefer to delay trading. This may arise because the investors are hoping for the jump in subjective expected value that occurs if the price increases by one cent (see Appendix A). Therefore, for individuals whose investments have gained or reduced in value, we might expect more trading on round numbers.

Figure 1 shows a visual representation of the model as applied in Figure 2 at the aggregate population level. The input stimulus is processed via analog processing and/or via percentage changes in value. We believe that, due to loss aversion, both these signals are increased in salience/strength if the choice involves loss. The relative final impact of these signals on aggregate choice preferences depends on the relative weighting/usage of each signal as well as the individual signal strengths themselves. We follow the established premise that judgment behavior is typically a probabilistic, rather than a deterministic, function of signal strength (Mosteller \& Nogee, 2006). As such, when signal strength is low due to weak/lowsalience signals or because of two salient but opposing signals, then more random errors will occur. When signal strength is strong/salient and both sources consistently point to the same, correct, choice then preference strength will be higher and fewer random errors will occur (a congruency effect).

There is no existing theory that explicitly predicts that losses should exaggerate the LDE and existing literature does not report evidence of this phenomenon. Therefore, if we do indeed 
observe that LDEs increase in loss situations, this will provide evidence in favor of our new analog value function and it will have revealed a new phenomenon, loss-induced LDEs.

\section{Hypotheses}

The LDE rests on the assumption that individuals have a tendency to judge changes in an investment's value based on analog heuristics (e.g., Eq. 2) rather than purely on the actual changes in value (e.g., Eq. 1). In fact, when changes in percentage values are not trivial to calculate, individuals may tend to rely more on left-digit heuristics. For example, consider an investor observing two assets that increased in value from $\$ 7,098$ to $\$ 7,987$ and from $\$ 7,204$ to $\$ 8,062$, respectively. The latter had increased by a lower percentage $(11.9 \%$ vs. $12.5 \%$ for the first asset) and yet, unlike the first asset, the leftmost digit of its value had increased (from 7 to 8). If the LDE influenced the investor's assessment then they might initially perceive the second price change as being more significant. Therefore, to confirm a LDE we first test the following hypothesis:

H1: Changes in the left-most digits of an investment's value will better predict preference than percentage changes in value.

We test this hypothesis via a controlled and counterbalanced laboratory experiment in which we ask participants to identify the best performing investment from pairs of investments. We manipulate changes in left digits of the investments' value and the percentage changes in value. The change in left digits is congruent with the actual change in value in half the trials. We employ logistic mixed effects regression analysis to determine to what extent the participants' choices of the better performing investments are predicted by the change in left digits of the investments' values (i.e. choices are driven by heuristic assessments; such as Eq. 2). This analysis controls for participants' preferences based on the actual percentage changes in value 
of these investments (i.e. preferences are driven by normative calculations, such as Eq. 1). If we do not observe any effect of the change in left digit on preferences then we can assume that $k=0$ in Eq. 2 and that left-digit heuristics are not used by participants. However, if the change in left digits is predictive of choice over-and-above the percentage change in investment value then we have evidence that $k>0$ and that participants are, to some extent, using a left-digit heuristic.

Most importantly, we also wish to test the veracity of the predictions of our analog value function (see Figure 2). Specifically, this predicts that when individuals are judging changes in values in the context of losses, the LDE will be greater. This prediction is based on the assumption that information will bias choices more if that information is more salient. Consequently, we also test hypothesis 2 :

H2: A change in the left digits of an investment's value when that investment has declined in value will have a greater impact on preferences than a change in left digits when that investment has gained in value.

We test Hypothesis 2 in our laboratory experiment, where participants face an equal number of decisions involving investments that have gained and investments that have decreased in value. If the predictions of our analog value functions are correct then we expect that the change in left-most digits of investments' values will better predict participants' choices of the better performing investments when they are assessing investments that have declined in value.

As illustrated in Figure 1, we expect, that both sources of information (changes in the left digit and changes in value) will play some role in choice behavior, but as long as the calculation of percentage changes is not trivial, individuals will tend to rely more on changes in left digits (i.e., we predict $.5<k<1$ ). We believe that loss aversion will increase the salience of the signals and that weak signals are less likely to influence choices. Therefore, in general, we 
should observe more random errors in the context of gains than in the context of losses, since gains do not benefit from the effect of loss aversion increasing signal salience. As the task is a binary forced choice, random errors in the context of gains will result in the probability of choosing either alternative being similar (i.e. $\operatorname{Pr}=1 / 2$ ).

We can also make error rate predictions with respect to the congruency of signals. In the event that the change in left digits is congruent with the actual change in percentage value, we would expect fewer errors as both signals point towards the correct choice. Combining these effects, we can predict that congruent-loss signals should result in lowest error rates as, in these conditions, salient/strong signals both point towards the same correct choice. We test this in Hypothesis 3:

H3: The lowest aggregate population error rate will occur in the congruent-loss condition.

To confirm the findings from our laboratory study, we use individual financial spreadtrader account data to test whether there is evidence of loss-induced LDEs in the form of the buy-sell imbalances on round numbers observed by Bhattacharya et al. (2012). Spread trading allows retail investors to speculate on the movement of underlying securities (e.g., indices, FX, shares, and commodities). No ownership of the underlying security takes place and traders who take a 'long' position gain/lose if the market rises/falls and those who take 'short' positions gain/lose if the market falls/rises. We give further details of the nature of these trades when discussing data in the section addressing the real-world trading method.

We employ the buy-sell ratio measure employed by Bhattacharya et al. (2012) to determine the buy-sell imbalance in the market:

$$
\text { Buy-Sell Ratio }=\frac{\text { Number of Buy Trades }}{\text { Number of Sell Trades }}
$$

Consider a spread trader who takes a 'long' position, but the market price subsequently falls, i.es/he is making a loss. We expect the investor to be more likely to realize that loss (i.e. sell 
their purchase ${ }^{4}$ ) when the market price is a round number. This arises because the analog value function predicts that a trader who is losing money would prefer to realize that loss atop one of the steps in the function (i.e. on a round number) rather than hold onto the position and face realizing at the bottom of the step (i.e. just below a round number). The drop from the top to the bottom of the step results in a sudden decrease in subjective value (see appendix A for an example vignette) and, as a result, we would expect more long trades in loss to be sold when the market price is on a round number. In other words, we predict significant over-selling of long positions in loss. Consequently, we will have a buy-sell imbalance in which the proportion of sell trades (of long positions) is greater when the market price is on a round number (i.e. Buy-Sell Ratio $<1$; indicating over-selling) than on another number (i.e. Buy-Sell Ratio $>1$; underselling).

Equally, we expect that investors with a long position in profit (i.e. the market price has risen) would prefer to wait until the price has risen atop a step (i.e. reached a round number) before they will realize their position (see Appendix A). This arises because the analog value function predicts a large increase in subjective expected value when the price rises to the point at the top of the step. If the price subsequently rises by one pricepoint there is only a marginal increase in subjective expected value. However, a reduction of one price point will result in a fall to the bottom of the step (i.e. a large decrease in subjective value). Consequently, we expect that the investor will prefer to realize their profit when the market price is on a round number. This preference to sell on round numbers should again result in the proportion of sell trades being greater when the market price is on round numbers (i.e. Buy-Sell Ratio $<1$; over-selling) than on other numbers (i.e. Buy-Sell Ratio > 1). In other words, we expect significant over-selling of long positions in gain.

There are similar predictions for 'short-sell' trades, except that the buy-sell imbalance will be the mirror image. When 'short selling' the investor 'buys' the market to realize profits and losses. Consequently, we should observe a greater proportion of buy trades when the 
market price is on round numbers (i.e. Buy-Sell Ratio $>1$; indicating over-buying) than on other numbers (i.e. Buy-Sell Ratio < 1; under-buying).

We examine these predictions relating to the behavior of spread traders by testing the following hypothesis:

H4: For long (short-sell) positions, the buy-sell ratio will be less than (greater than) one when the market price ${ }^{5}$ is on a round numbers and greater than (less than) one when it is on other numbers.

Most importantly, due to loss aversion, the step sizes for losses are larger than for gains, as illustrated in Figure 2. Thus, we expect the biasing effect of the LDE to be greater for losses than for gains and we expect greater buy-sell imbalances in the domain of losses (cf. gains). We examine this prediction by testing the following hypothesis:

H5: For both long and short-sell positions, the buy-sell imbalances will be greater (i.e. further from 1) for positions in loss than for positions in gain.

We test Hypotheses 4 and 5 by calculating the number of buy and sell trades at various price points for every trader in our real-world dataset. We then employ linear mixed effects modeling to predict the likelihood that a particular trader will buy or sell at certain round number pricepoints (i.e. ending in zero or five), depending on whether the trade is long or short and whether the trade relates to a position in profit or in loss.

\section{Laboratory Experiment Method}

\section{Participants}

Ninety-three postgraduate students, with a mean age of 23.98 (SD = 3.71) were recruited during a class break; 51 females and 42 males. Participants were not randomly 
selected, as only volunteers completed the task. No remuneration was offered since the task involved minimal time and effort. The booklets containing different condition permutations were shuffled before being handed out to ensure that conditions were randomly assigned to the volunteers.

\section{Materials and Procedure}

The survey took place in a lecture theatre in a break between classes. Participants were given a booklet with the information/instructions and a consent form on page one. In addition, they were verbally informed of the nature of the task. Those that wished to take part signed the consent form and were instructed not to confer with each other on answers. Participants then worked through the booklet. The second page contained an example trial. The following pages contained the testing trials, with one trial on each page. The order of pages was counterbalanced according to the design. The final page contained the debriefing information sheet.

Participants were tasked with choosing which of two assets had out-performed the other. Both assets had equal standard deviations of returns (see Table 1 for an example). A value-based decision should prefer the asset that returned the higher/lower percentage gain/loss. However, we suspect that participants may tend to choose the asset with a lower/higher percentage gain/loss if it has a larger increase/smaller decrease in the leftmost $\operatorname{digit}(\mathrm{s})$ and we suspect that this will be particularly pronounced when the choice involves assets that have decreased in value.

\section{[Table 1 approx. here]}

We presented participants with twelve forced choices between two assets, half had increased and half had decreased in value. We told the participants that these assets were all bought five months ago and, in the light of subsequent monthly price data provided, we asked them to identify the best performing investment from each pair. The best performing asset in each case was defined as the investment with the most favorable percentage change in value 
(since standard deviations were identical). We counterbalanced the order of assets between participants.

To assess the degree of LDE, we calculated the percentage change in the left digit of an asset's value. We achieved this by removing the digits to the right of the comma separator of the original (e.g., $£ 7,911)$ and final value of the asset $(£ 10,115)$ and we calculated the percentage change between the two resulting figures, i.e., $(10-7) / 7=43 \%$. The percentage change in value of the asset that increased most/decreased least in value was congruent with the change in left digits in only half the gain choices and half the loss choices. In the other half of choices, the change in leftmost digits was incongruent with the best performing alternative (see Table 2). For example, in the fifty percent of choices involving gains, the asset that returned the greatest gain, also exhibited the greatest increase in the left digit of its price (i.e. congruent-gain condition). In the other fifty percent of the choices involving gains, the asset that returned the least gain, exhibited the greatest increase in the left digit of its price (incongruent-gain condition).

[Table 2 approx. here]

\section{Models}

We estimated models to determine the factors that influenced the participants' choices of the best performing asset. In these models we defined the dependent variable, Choice $_{i j}$, as the choice of asset A/B (coded 1/0) for trial $i$ for participant $j$. We defined a 'value-based choice variable', $\Delta$ Value $_{i}$, as $V\left(A_{i}\right)-V\left(B_{i}\right)$, where $V\left(x_{i}\right)$ represents the percentage change in asset $x_{i}^{\prime} \mathrm{s}$ value in trial $i$; a positive/negative $\Delta$ Value $_{i}$ indicates that $A_{i} / B_{i}$ should be preferred (based on a higher-level numerical processing strategy). Similarly, we defined a 'left-digit heuristic' variable, $\Delta$ LeftDigits $_{i}$, as $D\left(A_{i}\right)-D\left(B_{i}\right)$, where $D\left(x_{i}\right)$ represents the percentage change in the leftmost $\operatorname{digit}(\mathrm{s})$ of asset $x_{i}^{\prime}$ s value in trial $i$, so that a positive/negative $\Delta$ LeftDigits $i$ indicates a preference (based on a heuristic, analog system, strategy) for $A_{i} / B_{i}$. To test the degree to which loss 
aversion affects the LDE, we introduced a dummy variable, $\operatorname{Loss}_{i}$, which took the value of $0 / 1$ when a pair of assets increased/decreased in value.

We estimated the extent that the value-based $\left(\Delta\right.$ Value $\left._{i}\right)$ or analog-heuristic strategy $\left(\Delta\right.$ LeftDigits $_{i}$ ) better accounted for participants' choices (Hypothesis 1) and if the effects were influenced by whether the decision involved assets which had gained or reduced in value (Hypothesis 2). In order to correctly control for the repeated-measures design, we employed logistic mixed-effects models to account for participants' choices of assets, allowing the intercept to vary depending on a fitted random coefficient $b_{j}$ for participant $j$.

We also estimated participants' reliance on the left-digit heuristic by estimating the parameters of the value function shown in Equation 6a (see below). Note that this equation is representative of the model illustrated in Figure 1. To do this, we calculated the proportion of alternatives A (vs. B) selected by participants and searched for the parameter values (i.e. $k, \alpha, \beta$ and $\lambda$ ) that resulted in the best fit to this aggregated choice behavior. We estimated nonlinear least-squares estimates of the parameters in the following non-linear model, via a grid search algorithm:

$$
\begin{aligned}
& P(A, B)_{l}=L\left(A_{\text {Original }_{l}} A_{\text {Present }_{l}}, B_{\text {Original }_{l}}, B_{\text {Present }_{l}}, k, \alpha, \beta, \lambda\right)+\varepsilon_{l}, \\
& \text { where, } L=v\left[h\left(\frac{A_{\text {original }_{l}}}{1000}, \frac{A_{\text {Present }}}{1000}, k\right)\right]-v\left[h\left(\frac{B_{\text {Original }}}{1000}, \frac{B_{\text {Present }_{l}}}{1000}, k\right)\right] .
\end{aligned}
$$

The function $h$ is the left-digit heuristic (see Eq. 2) applied to the present (i.e. end of month 5 in the laboratory experiment) and original values of assets A and B divided by 1000 for choice set $l$ (where $l=1,2, \ldots, 12$, see Table 2 ). The assets were divided by 1000 since, as discussed above, we expect the salient left-digit integers to be those to the left of the thousands comma separator. The value function, $v$, is the value function applied in prospect theory (see, Eq. 4) for which the parameters $\alpha, \beta$ and $\lambda$ were estimated. Note that the response variable, $\left(P(A, B)_{l}\right)$, was normalized with the center defined as 0.5 and the result of $L\left(A_{\text {Original }_{l}}, A_{\text {Present }_{l}}, B_{\text {Original }_{l}}, B_{\text {Present }_{l}}, k, \alpha, \beta, \lambda\right)$ was normalized with the center defined as 
zero. We repeated this process to estimate the parameters for the traditional prospect theory value function, whereby $L$ is replaced with $T$, such that:

$$
\begin{aligned}
& P(A, B)_{l}=T\left(A_{\text {Original }_{l}}, A_{\text {Present }_{l}}, B_{\text {Original }_{l}}, B_{\text {Present }_{l}}, \alpha, \beta, \lambda\right)+\varepsilon_{l}, \\
& \text { where, } T=v\left[d\left(A_{\text {Present }_{l}}, A_{\text {Original }_{l}}\right)\right]-v\left[d\left(B_{\text {Present }_{l}}, B_{\text {Original }_{l}}\right)\right] .
\end{aligned}
$$

Once we had estimated these functions, we used them as independent variables in two separate logistic mixed-effects regression models that were designed to predict participants' choices (i.e. Choice ${ }_{i j}$ ). In other words, each model only included the relevant decision rule function (i.e. $T$ or $L$ ) and a random factor allowing the intercept to vary by participant $j$. We expected that the model using the analog value function would better explain participants' choices than the model using the traditional value function from prospect theory. We tested this expectation by examining whether the model using the analog value function had a lower AIC and BIC and by employing Cox tests for non-nested models.

\section{Results and Discussion}

Table 2 shows the percentage of participants who chose asset A over B in each trial. We estimated the following mixed effects logistic regression model using the choice data:

$$
\text { Choice }_{i j}=\beta_{0}+\beta_{1} \Delta \text { Value }_{i}+\beta_{2} \Delta \text { LeftDigits }_{i}+\beta_{3} \text { Loss }_{i}+\beta_{4}\left(\Delta \text { LeftDigits }_{i} \times \text { Loss }_{i}\right)+b_{j}+\varepsilon_{i j}
$$

The results are displayed in Table 3 and demonstrate that the inclusion of $\Delta$ Value $_{i}$ was not a significant factor in predicting choices. Indeed, the removal of $\Delta V a l u e_{\mathrm{i}}$ from Eq. 1 did not significantly reduce model fit $\left(\triangle \mathrm{AIC}=1.9\right.$, log-likelihood ratio $\left.(\mathrm{LLR}) \chi^{2}(1)=0.12, p=.733\right)$. However, the significant main effect of $\Delta$ LeftDigits $_{i}$ suggests that a change in left digit did affect choice behavior; in line with Hypothesis 1. In addition, the significant interaction $\left(\Delta\right.$ LeftDigits $_{i} \times$ Loss $\left._{i}\right)$ indicated that the LDE depended on whether the choice involved gains or losses; in line with Hypothesis 2. The two hypotheses were further confirmed by the fact 
that the Phi correlation coefficient, $\phi^{2}$, between $\Delta$ Value $_{i}$ (rational strategy) and choice behavior was only .03, whereas, that between $\Delta$ LeftDigits $_{i}$ (heuristic strategy) and choice behavior was .14, indicating a greater reliance on analog numerical heuristics (cf. higherlevel reasoning).

[Table 3 approx. here]

To explore the significant interaction between $\Delta$ LeftDigits $_{i}$ and Loss $_{i}$, we estimated separate models of the following form for assets that increased $(l=0)$ and decreased $(l=1)$ in value:

$$
\text { Choice }_{i j l}=\beta_{0}+\beta_{1} \Delta \text { LeftDigits }_{i l}+\beta_{2} \Delta \text { Value }_{i l}+b_{j}+\varepsilon_{i j}
$$

For assets that decreased in value (losses), the $\Delta$ LeftDigits $_{i}$ variable was significant $\left(\Delta \mathrm{AIC}=20.56, \log\right.$-likelihood ratio $\left.(\mathrm{LLR}) \chi^{2}(1)=22.57, p<.001, \phi^{2}=.20\right)$ whereas the $\Delta$ Value $_{i}$ factor was not $\left(\triangle \mathrm{AIC}=1.97, \log\right.$-likelihood ratio $\left.(\mathrm{LLR}) \chi^{2}(1)=0.03, p=.859, \phi^{2}=.07\right)$. This suggests that the change in the left digits was a significant factor influencing choices involving loss. For assets that increased in value, neither the $\Delta$ LeftDigits $_{i}$ factor $\left(\Delta \mathrm{AIC}=.05\right.$, LLR $\chi^{2}(1)=$ $\left.1.95, p=.163, \phi^{2}=.08\right)$ nor the $\Delta$ Value $_{i}$ variable $\left(\Delta \mathrm{AIC}=1.81, \log\right.$-likelihood ratio $(\mathrm{LLR}) \chi^{2}(1)=$ $0.185, p=.667, \phi^{2}=-.01$ ) were significant (i.e. when added successively to a model which only included $\beta_{0}$ and $b_{j}$ they added no predictive information). This indicates that for gains, neither changes in LDE nor percentage changes in value appeared to predict choices significantly. This finding is consistent with the idea that only weak signals are produced in gains, as these signals are not afforded the salience of similar changes in loss. This, therefore, may result in more random errors. However, for assets that decreased in value, the impact of the change in left digits was sufficient to influence choices significantly. In summary, our results are consistent with the application of our analog value function (Figure 2), which predicts that the LDE will have a much greater biasing effect in the domain of loss.

The results of the nonlinear least squares grid-search estimation of the analog value function applied to the participant's aggregate data are shown in Table 4. Notably, the estimation for the left-digit heuristic weighting parameter was greater than zero $(k=.56)$, 
suggesting that the left-digit heuristic was being employed. Our analog value function better explained participants' choices than the traditional value function model. This was confirmed by the lower AIC $(\triangle \mathrm{AIC}=8.6)$ and BIC $(\triangle \mathrm{BIC}=8.6)$ statistics for the analog value function (cf. traditional value function) logistic mixed-effects model (Table 4). It was also confirmed by the fact that the Cox tests for the models employing the analog value function and the traditional value function were significant and insignificant, respectively.

[Table 4 approx. here]

It should be noted that the parameters estimated for the traditional prospect theory model are atypical when compared to values from studies designed to determine these values (see Fox \& Poldrack, 2009; for a review). The fit for the analog value function model, on the other hand, is much more typical. We suspect that this may have arisen because the prospect theory fit relies on individuals perceiving the change in the assets value in a conventional, nonheuristic, fashion. However, if participants were, as we expected, employing an analog heuristic it is not surprising that it is difficult to establish reliable parameters using the traditional value function from prospect theory. Once we fit the model including the analog value function, the prospect theory parameters appear to converge to levels that are more in line with previous studies.

Since the estimated analog weighting parameter $(k=0.56)$ is less than one and greater than 0.5 , our model accounts for our results by allowing both analog heuristic information and percentage change information to affect choices to some extent. However, the analog heuristic tends to be more heavily weighted. Given this, the model would predict greater preference strength in the congruent (cf. incongruent) situations and, in particular, in loss situations (see Hypothesis 3). Figure 3 displays the results concerning the effect of congruency between the change in left digits and the percentage change in value on the error rate, where an error was defined as a participant selecting the alternative that had increased/decreased in value by the least/most. The results show that there was indeed a lower error rate in congruent conditions 
when the change in left-digits coincided with the change in value $(t(1110.9)=-4.56, p<.001)$. In support of Hypothesis 3, the effect was greater in loss trials. The error rate was significantly lower in the congruent-loss trials compared to the incongruent-loss trials $(t(552.31)=-4.72, p<$ .001). However, for congruent-gain trials, the reduction in error rate was only marginally significant when compared to error rate in the incongruent-gain trials $(t[555.98)=-1.78, p=$ .075). In other words, particularly in loss, when the change in left-most digits coincides with the actual change in value of the investments, individuals tend to more accurately select the better performing investment. However, when the change in left-most digits does not coincide with the best performing asset, they appear to be biased by this heuristic and are less likely to accurately select the best performing asset.

[Figure 3 Approx. here]

This result confirms the linear mixed model findings, namely, that the biasing effect of the left-digit heuristic on the ability to judge the best performing asset is greater in judgments involving loss (cf. gain). Our model predicted this effect and explains it via an increased salience of left-digit changes when the changes are associated with judging losses.

\section{Real-World Trading Method}

In order to test Hypotheses 4 and 5, we examined whether the degree of buy-sell imbalance observed in real-world trading data was different in situations where the trading decisions involved gains or losses.

\section{Data}

The data were supplied by a large UK retail spread-trading brokerage and contained 8,441,805 individual trades made by 28,211 individuals, trading in the DAX 30 and FTSE 100 Rolling Daily Indices and the GBP/USD and EUR/USD Rolling Daily currency pairs. Spread trading allows retail investors to speculate on the movement of underlying securities (e.g., 
indices, FX, shares, and commodities). No ownership of the underlying security takes place and any profits secured are not subject to capital gains tax. Positions are highly geared, meaning that individuals can leverage large positions with relatively small capital6. The main advantage of this data is that it contains a record of all transactions for each position held by each individual. This enables us to determine the exact execution prices and to calculate, with precision, the profits and losses incurred. In addition, given that the retail sector participants are not necessarily professional investors, we are more likely to be able to measure LDEs, since less informed investors tend to be more susceptible to cognitive bias (Dhar \& Zhu, 2006). The data involves trades conducted between $8^{\text {th }}$ November 2004 and the $31^{\text {st }}$ of March 2013. For each trade we establish its execution time and price, whether it was a 'buy' or 'sell' trade for a long or short-sell position (see Footnote 2) and the profit/loss made on each position.

Bhattacharya et al. (2012) examined the transactions of stocks priced in dollars (i.e. that have an integer to the left of a decimal point and cents to the right, e.g., $\$ 1.99, \$ 4.74$, etc.) from the New York Stock Exchange (NYSE) and Quote (TAQ) data set. Spread-trading data does not come in this form. Consequently, in order to make our dataset more comparable, we first converted the raw prices in each market into point-based prices, which are those employed by spread traders. We achieve this by dividing the raw prices by the market's 'pip size'. For example, a typical price for the GBP/USD pair may be 1.6481. However, the number to the left of the decimal point is not interesting to investors as it is highly unlikely to change. Rather, investors look at converted prices based on the pip size, which is .0001 for this pair (EUR/USD also has a pip size of .0001 and the indices DAX 30 and FTSE 100 Rolling Daily both have a pip size of 1). Therefore, the point-converted price is 16481 and a rise to 16482 would result in a $\$ 10$ gain for someone who had taken a $\$ 10$ long position in the GBP/USD. By converting all assets to point-based prices, we can easily compare the indices and foreign exchange pairs together. Therefore, for all point-converted prices a change in the right-most digit by one equates the same gain/loss per unit staked in a long or short position. Given the manner in 
which the point-converted prices and stock prices change under normal market conditions, we anticipate that spread traders and stock-exchange traders, respectively, will view the last two digits in point-converted prices and the two digits to the right of the decimal place in stock prices, similarly (such as those explored in Bhattacharya et al.'s (2012) study). In addition, we anticipate that spread traders will view the third last digit in the point-based prices in a similar manner to investors when viewing the first integer to the left of the decimal point in stock prices. We believe that this is a reasonable assumption since it is very rare for the fourth digit from the right hand end of the points-converted prices to change. In fact, in our data, there were only 116,306 trades $(1.38 \%)$ in which the first digit to change was beyond the third from last digit and we excluded these extreme cases from analysis. This left 8,325,499 trades for analysis. Therefore, in our results we simply refer to the last three digits of the point-converted price as the 'point-price'. For example, a GBP/USD price of 1.6481 has a point-converted price of 16481 and a 'point-price' of 481; where 4 and 1 are what we expect investors would perceive as the leftmost and rightmost digit integers, respectively.

Bhattacharya et al. (2012) discovered that buy-sell imbalances could also arise from a cause other than the LDE. In particular, they found buy-sell imbalances above and below round numbers, rather than on round numbers. This they attributed to a 'cluster undercutting effect,' resulting from individuals competing in the market setting their limit orders. This type of buysell imbalance is not the focus of our study, as we are only interested in the buy-sell imbalances that occur due to the LDE. Consequently, to eliminate any 'cluster undercutting effects', we confined our analysis to market order transactions ${ }^{7}$, in other words, excluding all limit-order transactions. This resulted in us removing 768,779 transactions that were not market order transactions, leaving 7,571,611 transactions for analysis. Since each transaction involves a buy and a sell trade, this left 15,143,222 trades for analysis, half of which were buy and half were sell trades. 
In order to test our hypotheses we estimated a logistic mixed effects model with an individual trader's likelihood of taking a long or short position as the dependent variable. The independent variables in this model included: (1) Short, coded 0 or 1 for long or short positions, respectively; (2) Loss, coded 0 or 1 for trades closed in gain or loss, respectively; (3) Zero, coded 1 whether the last digit of the trade price ended in a zero, and zero otherwise; (4) Five, coded 1 if the last digit of the trade price ended in five, and zero otherwise. We also included interaction terms to model the expected inversion of the buy-sell imbalances between long and short positions (i.e. Five $\times$ Short and Zero $\times$ Short), as outlined in the hypotheses section. We also included interaction terms to model the expected increased buy-sell imbalance associated with losses (i.e. Five $\times$ Loss, Zero $\times$ Loss, Short $\times$ Loss, Five $\times$ Short $\times$ Loss and Zero $\times$ Short $\times$ Loss) .

Consequently, the model we estimated was as follows:

$$
\begin{gathered}
P(\text { Buy }, \text { Sell })_{i j}=f\left\{\beta_{0}+\beta_{1} \text { Five }_{i j}+\beta_{2} \text { Zero }_{i j}+\beta_{3} \text { Short }_{i j}+\beta_{4} \text { Loss }_{i j}+\beta_{5}(\text { Five } \times \text { Short })_{i j}+\right. \\
\beta_{6}(\text { Zero } \times \text { Short })_{i j}+\beta_{7}(\text { Five } \times \text { Loss })_{i j}+\beta_{8}\left(\text { Zero } \times \text { Loss }_{i j}+\beta_{9}(\text { Short } \times \text { Loss })_{i j}+\right. \\
\left.\beta_{10}(\text { Five } \times \text { Short } \times \text { Loss })_{i j}+\beta_{11}(\text { Zero } \times \text { Short } \times \text { Loss })_{i j}+b_{j}+\varepsilon_{i j}\right\}
\end{gathered}
$$

where $i$ indicates the trade executed by trader $j, \beta_{n}$ indicates the fixed effects coefficient and $b_{j}$ is the estimated random coefficient to control for the individual variation.

\section{Results and Discussion}

\section{Buy-Sell Imbalances on Round Numbers}

We first examined whether there were buy-sell imbalances at any particular rightmost digit of the point prices. We did this by plotting buy-sell ratios (Eq. 5) at different final two digits of point-prices, from X00 to X99. We present these plots in Figure 4 for long and short positions realized in loss or in profit. This enables comparison to previous findings from Bhattacharya et al. (2012).

[Figure 4 approx. here] 
Our analog value function suggested that the realization price of a gain or loss would occur more on round numbers. Recall that for long/short positions the realization trade is a sell/buy trade (see Footnote 2). Therefore, for long positions, we expected more over-selling (i.e. Buy-Sell Ratio $<1$ ) on round numbers and, therefore, under-selling (i.e. Buy-Sell Ratio $>$ 1) on other numbers. Equally, for short positions we expected the opposite pattern; more over-buying (i.e. Buy-Sell Ratio $>$ 1) on round numbers and under-buying (i.e. Buy-Sell Ratio $<1$ ) on other numbers. This, in fact, is the pattern we observe and, as expected, the pattern is most clearly observable associated with positions in loss. In short, the plots in Figure 4 appear to support Hypothesis 4, namely, that we observe characteristic buy-sell imbalances on round numbers, i.e. those ending in zero (XX0) and five (XX5). However, we do not observe such a strong pattern for positions that have gained in value. In particular, there appears to be a little over-selling (BuySell Ratio $<1$ ) of long positions on round numbers ending in zero for positions in profit, but the pattern is not nearly as clear as it is for losses. If the buy-sell imbalance were indeed significantly greater for positions in loss then this would support Hypothesis 5 . The finding would also indicate that our experimental findings extend to real-world situations.

\section{Analysis at the individual level}

The observations made above are more apparent when we examine the mean of all clients' individual buy-sell ratios at each final digit of the price-point (see Figure 5). We summed the number of buy and sell trades placed by each trader at each price-point, based on the last digit of each price-point. We then plotted the mean of all the traders' buy-sell ratios together with 95\% confidence interval error bars (see Figure 5). Table 5 compares the buy-sell ratios for just the price-points ending in zero, five or any other ending for long and short positions realized in profit or in loss.

[Figure 5 and Table 5 approx. here] 
The pattern in both Figure 5 and Table 5 is clear. As predicted, for long positions there appears to be more over-selling (Buy-Sell Ratio < 0) on price-points ending in five and zero and under-selling on other price-points. Equally, for short positions there appears to be more overbuying (Buy-Sell Ratio $>$ 0) on price-points ending in five and zero than on other numbers. In order to test the significance of this effect, we analysed the data using the logistic mixed effects model shown in Eq. 10. This controls for the repeated-measures nature of our data by allowing the intercept to vary for each individual. The estimated coefficients of this model are presented in Table 6.

[Table 6 approx. here]

We first examine the results relating to long positions. The coefficients displayed in Table 6 indicate that for long positions in profit, there are significantly more sells than buys on price-points ending in five $(z=21.74, p<.001)$ and ending in zero $(z=18.01, p<.001)$. This imbalance on round numbers is consistent with Hypothesis 4. For long positions in loss there were, again, significantly more sells than buys on price-points ending in five $(z=20.8, p<.001)$ and those ending in zero $(z=67.08, p<.001)$. Interestingly, this buy-sell imbalance towards over-selling long positions in loss was greater on prices ending in zero than ending in five $(z=$ 31.59, $p<.001$ ). Most importantly, and consistent with Hypothesis 5, the buy-sell imbalance on round numbers in long positions was greater in loss than for gains in both price-points ending in zero $(z=40.52, p<.001)$ and five $(\mathrm{z}=2.70, p=.007)$.

The results relating to short positions were also consistent with Hypothesis 4. In particular, for short positions in gain, there were significantly more buys than sells on prices ending in five $(z=3.30, p<.001)$ and zero $(z=11.82, p<.001)$. This imbalance was greater for short positions closed for a gain on price-points ending in zero than for those ending in five $(z=$ 5.90, $p<.001$ ). For short-sell positions closed in loss, again consistent with Hypothesis 4, there were more buys than sells on prices ending in zero $(z=46.51, p<.001)$ and five $(z=24.35, p<$ .001). This imbalance in short-sell positions closed in loss was again larger for price-points 
ending in zero than in five $(z=14.91, p<.001)$. Most importantly, consistent with Hypothesis 5 , for short positions, the imbalance towards buying (cf. selling) on round numbers was greater for positions in loss than for positions in gain for price-points ending in zero $(z=28.81, p<.001)$ and five $(z=16.95, p<.001)$.

In summary, these results support Hypothesis 4, as we observe significantly greater selling of long positions and buying of short positions on round numbers. In addition, in support of Hypothesis 5, we observe significantly greater buy-sell imbalances on round numbers for positions in loss (cf. gain).

\section{General Discussion}

Our results confirm that there are robust biases towards round numbers and that this bias is greater when the decision involves losses. The effect of loss exacerbating the impact of a change in left digits was predicted by the analog value function we proposed. This function was the outcome of applying analog numerical processing during the editing phase of prospect theory. The function displays three key characteristics: (1) subjective expected value has clear jumps when the left digit changes; (2) due to loss aversion, the LDE (described by the size of the step) is larger in the domain of loss (cf. gains); (3) the size of the LDE depends on the individual's propensity to use analog heuristics in general and, also, whether the context of the choice (e.g. time-pressure, task complexity, low cost of a poor decision) elicits greater reliance on analog heuristics (measured by parameter $k$ in eq. 2).

In support of the first of these key characteristics, our laboratory study demonstrated a tendency to be biased by the LDE when assessing the change in hypothetical investment values. Furthermore, our analysis of real-world trading data reveals robust buy-sell imbalances occurring on round numbers. In support of the second of these key characteristics, we observed in both the laboratory study and in the trading data analysis, greater left-digit biases in the domain of loss than in the domain of gains. These findings support the view that decision- 
dependent emotions play a key role in the behavioral anomalies observed to impact economic decision-making (Summers \& Duxbury, 2012). We did not test the third key characteristic explicitly in our study. However, our estimation of the left-digit heuristic parameters suggested that $k=.56$, which indicates a significant impact of the change in left-digits on the perception of change in value. We estimated this based on analysis of aggregate data. Consequently, we did not determine the amount of variation between individuals in $k$ nor did we investigate the extent to which the context of the decision may affect $k$. These are both exciting future directions for assessing the nature and characteristics of the left-digit heuristic in biasing choices. For example, recent studies suggest that certain individuals are more influenced by round numbers and indeed this appears to affect their profitability (see, Kuo et al., 2014). It would be interesting to measure this effect by estimating $k$ for different populations and indeed for the same individuals in different tasks varying in complexity, time-pressure and cost of error.

Our findings pose a problem for some of the conclusions drawn by Kuo et al. (2014). In the paper, the authors concluded that cognitive limitation may be manifested in a tendency to over-trade on round numbers. Kuo et al. (2014) then observed that there was a positive correlation between an individual's tendency to trade on round numbers and their underperformance. However, our results suggest that those traders who tend to close more trades in loss (i.e. those that are poor performers for whatever reason) would naturally tend to close more positions on round numbers compared to those that tend to close more winning trades. Of course, both explanations are not mutually exclusive. It is possible that cognitive limitation leads to greater execution of trades on round numbers but also that loss-exacerbated round-number bias occurs as well. Nevertheless, our findings suggest that correlation between the extent of round number bias and investor performance cannot only be explained by cognitive limitation. 
An important potential limitation of our laboratory study is that we did not incentivize the participants. Consequently, they may have been more likely to rely on analog heuristics than they might in a real-world investment decision. Indeed, as we discussed when introducing our analog value function, in a properly incentivised task, we do not expect to observe such a striking use of the left-digit heuristic. In other words, we expect an incentivised context to reduce $k$ closer towards zero. Nevertheless, this does not explain why we observe significant differences in the impact of the left-digit bias associated with winning and losing investments. Moreover, given that we observe similar loss exacerbation of the LDE in our analysis of realworld data, where individuals are making decisions regarding real money, we are convinced that our results provide clear evidence that losses do exacerbate the LDE. However, we do expect that the degree to which the LDE affects choices may vary depending on the individual and the context of the choice. An important consideration is that our real-world analysis used data from the UK retail spread-trading market. This population is made-up of individual investors from the retail sector who invest relatively small amounts when compared with institutional investors. Nevertheless, our aim in this paper was to test for a psychological explanation for LDEs and whether or not, therefore, loss-aversion might exacerbate this bias. For this reason, retail spread-traders are a particularly good group to examine, since Kuo et al. (2014) have demonstrated that individual investors are those more prone (cf. institutional investors) to the LDE. Therefore, we expect that the LDE observed in this population will be higher than that for more informed participants in wider financial markets. However, based on the findings of other research into LDEs in wider financial markets (e.g., Bhattacharya et al., 2012; Kuo et al., 2014), we can be certain that even in these more traditional markets, $k$ is unlikely to be zero. Therefore, it is extremely likely that even in traditional financial markets we would observe the kind of loss-induced LDE we found in our analyses, even if the overall effect size is smaller. 


\section{Conclusion}

We discovered that decisions regarding losses are more affected by the LDE than decisions regarding gains. This conclusion was reached based on results from a laboratory study and analysis of individual accounts from a real-world market. We capture the increased biasing effect of the left-digit in the domain of loss by our proposed analog value function. This function was the result of applying analog numerical heuristics during the editing phase of prospect theory.

Beyond the discovery of loss-induced LDEs, our results are important for prospect theory in general because they reveal the importance of the editing phase in modeling decisions. Far more attention has been paid to prospect theory's evaluation stage in the literature but our results reveal that certain heuristics (e.g. analog numerical heuristics) applied in the editing phase can significantly impact both prospect theory predictions, as well as the accuracy of the estimation of its parameters from observed data. In addition to improving prospect theory predictions, the analog value function proposed in this paper also provides a way of measuring the extent that an individual may suffer from LDEs. The $k$ parameter (in Equation 2) can be estimated for individuals to determine the extent to which they tend to rely more on analog (vs. higher order numerical) processing, as well as estimating the effect of certain situational factors (e.g. task complexity, time-pressure, etc.) on the use of analog heuristics.

Finally, given the potential negative financial consequences for those investors susceptible to this bias (see, Bhattacharya et al., 2012), interventions should be considered in designing decision support systems. Such interventions could attempt to determine an investor's propensity to employ left-digit heuristics (i.e. estimating the $k$ parameter for a particular individual) and those who are particularly vulnerable could receive additional training in how to deal with this bias when under pressure and making decisions regarding losing investments. 


\section{References}

Allen, E., Dechow, P., Pope, D., \& Wu, G. (2015). Reference-dependent preferences: Evidence from marathon runners. Manuscript submitted for publication.

Ahn, H. J., Cai, J., \& Cheung, Y. L. (2005). Price clustering on the limit-order book: Evidence from the Stock Exchange of Hong Kong. Journal of Financial Markets, 8(4), 421-451.

Basu, K. (1997). Why are so many goods priced to end in nine? And why this practice hurts the producers. Economics Letters, 54, 41-44.

Bhattacharya, U., Holden, C. W., \& Jacobsen, S. (2012). Penny wise, dollar foolish: Buy-sell imbalances on and around round numbers. Management Science, 58(2), 413-431.

Brannon, E. M. (2002). The development of ordinal numerical knowledge in infancy. Cognition, $82,223-240$.

Brannon, E. M., \& Terrace, H. S. (1998). Ordering of numerositites 1 to 9 by monkeys. Science, 282, 746-246.

Busse, M. R., Lacetera, N., Pope, D. G., Silva-Risso, J., \& Sydnor, J. R. (2013). Estimating the effect of salience in wholesale and retail car markets. American Economic Review: Papers and Proceedings, 103, 575-579.

Chiao, C., \& Wang, Z. M. (2009). Price clustering: Evidence using comprehensive limit-order data. Financial Review, 44(1), 1-29.

Dhar, R., \& Zhu, N. (2006). Up close and personal: Investor sophistication and the disposition effect. Management Science, 52(5), 726-740.

Evans, J. S. B., \& Stanovich, K. E. (2013a). Dual-process theories of higher cognition advancing the debate. Perspectives on Psychological Science, 8(3), 223-241.

Evans, J. S. B., \& Stanovich, K. E. (2013b). Theory and metatheory in the study of dual processing reply to comments. Perspectives on Psychological Science, 8(3), 263-271. 
Fox, C. R., \& Poldrack, R. A. (2009). Prospect theory and the brain. Neuroeconomics: Decision making and the brain, 145, 174.

Fraser-Mackenzie, P.A.F., \& Dror, I.E. (2011). Dynamic reasoning and time pressure: Transition from analytical operations to experiential responses. Theory and Decision. 71(2), 211225.

Gordon, P. (2004). Numerical cognition without words: Evidence from Amazonia. Science, 306, 496-499.

Harris, L. (1991). Stock price clustering and discreteness. Review of Financial Studies, 4(3), 389415.

Hauser, M. D., Carey, S., \& Hauser, L. B. (2000). Spontaneous number representations in semifree-ranging rhesus monkeys. Proceedings from the Royal Society of London, B: Biological Sciences, 267, 829-833.

Hinrichs, J. V., Yurko, D. S., \& Hu, J. M. (1981). Two-digit number comparison: Use of place information. Journal of Experimental Psychology: Human Perception and Performance, 7, 890-901.

Hogarth, R. M. (2001). Educating Intuition. Chicago, IL: University of Chicago Press.

Ikenberry, D. L., \& Weston, J. P. (2008). Clustering in US stock prices after decimalisation. European Financial Management, 14(1), 30-54.

Kahneman, D., \& Frederick, S. (2002). Representativeness revisited: Attribute substitution in intuitive judgement. In T. Gilovich, D. Griffin, \& D. Kahneman (Eds.), Heuristics and biases: The psychology of intuitive judgment (pp. 49-81). Cambridge, MA: Cambridge University Press.

Kahneman, D., \& Tversky, A. (1979). Prospect theory: An analysis of decision under risk. Econometrica, 263-291. 
Keren, G. (2013). A tale of two systems: A scientific advance or a theoretical stone soup? Commentary on Evans \& Stanovich (2013). Perspectives on Psychological Science, 8(3), 257-262.

Kruglanski, A. W. (2013). Only one? The default interventionist perspective as a unimodelCommentary on Evans \& Stanovich (2013). Perspectives on Psychological Science, 8(3), 242-247.

Kruglanski, A. W., \& Gigerenzer, G. (2011). Intuitive and deliberate judgments are based on common principles. Psychological Review, 118(1), 97.

Kuo, W. Y., Lin, T. C., \& Zhao, J. (2014). Cognitive limitation and investment performance: Evidence from limit order clustering. Review of Financial Studies, 28(3), 838-875.

Lacetera, N., Pope D. G., \& Syndor, J. R. (2012). Heuristic thinking and limited attention in the car market. American Economic Review, 102, 2206-2236.

Lemer, C., Dehance, S., Spelke, E., \& Cohen, L. (2003). Approximate quantities and exact words: dissociable systems. Neuropsychologica, 41, 1942-1958.

Markle, A., Wu, G., White, R., \& Sacket, A. (2015). Goals as reference points in marathon running: A novel test of reference-dependence, Manuscript submitted for publication.

Manning, K. C., \& Sprott, D. E. (2009). Price endings, left-digit effects, and choice. Journal of Consumer Research, 36(2), 328-335.

Monroe, K. B. (2003). Pricing: Making profitable decisions, New York: McGraw-Hill/Irwin.

Mosteller, F., \& Nogee, P. (2006). An experimental measurement of utility. In Selected Papers of Frederick Mosteller (pp. 179-219). Springer New York.

Niederhoffer, V. (1965). Clustering of Stock Prices. Operations Research, 13, 258-265.

Niederhoffer, V. (1966). A new look at the clustering of stock prices. Journal of Business, 39, 309313.

Payne, J. W., Bettman, J. R., \& Johnson, E. J. (1993). The adaptive decision maker. Cambridge University Press. 
Pennycook, G., Fugelsang, J. A., \& Koehler, D. J. (2015). What makes us think? A three-stage dualprocess model of analytic engagement. Cognitive psychology, 80, 34-72.

Pica, P., Lemer, C., Izard, V., \& Dehaene, S. (2004). Exact and approximate arithmetic in an Amazonian indigene group. Science, 306, 499-503.

Poltrock, S. E., \& Schwartz, D. R. (1984). Comparative judgements of multidigit numbers. Journal of Experimental Psychology: Learning, Memory, and Cognition, 10, 32-45.

Sloman, S. A. (1996). The empirical case for two systems of reasoning. Psychological Bulletin, $119,3-22$.

Stanovich, K. E., \& West, R. F. (2000). Individual differences in reasoning: Implications for the rationality debate. Behavioral and Brain Sciences, 23, 645-726.

Stiving, M. (2000). Price-endings when prices signal quality. Management Science, 46(12), 16171629.

Stiving, M., \& Winer, R.S. (1997), An empirical analysis of price endings with scanner data, Journal of Consumer Research, 24, 57-67.

Summers, B., \& Duxbury, D. (2012). Decision-dependent emotions and behavioral anomalies. Organizational Behavior and Human Decision Processes, 118(2), 226-238.

Thomas, M., \& Morwitz, V. G. (2005). Penny wise and pound foolish: The left-digit effect in price cognition. Journal of Consumer Research, 32(1), 54-64

Tversky, A., \& Kahneman, D. (1974). Judgment under uncertainty: Heuristics and biases. Science, $185,1124-1131$.

Tversky, A., \& Kahneman, D. (1991). Loss aversion in riskless choice: A reference-dependent model. The Quarterly Journal of Economics, 106(4), 1039-1061.

Tversky, A., \& Kahneman, D. (1992). Advances in prospect theory: Cumulative representation of uncertainty. Journal of Risk and Uncertainty, 5(4), 297-323.

Wynn, K., Bloom, P., \& Chiang, W. C. (2002). Enumeration of collective entities by 5-month-old infants. Cognition, 83, 55-62. 


\section{Footnotes}

${ }^{1}$ Instructions to a broker to buy/sell securities once the market reaches a given price.

${ }^{2}$ They also observed excess buying/selling just below and above round numbers. This appeared to be the result of investors attempting to ensure deals are made by undercutting other bidders in the market that they suspect have orders on the round numbers; an effect called 'limit order undercutting'.

${ }^{3}$ This is a very simple version of an analog heuristic function for explanatory purposes. This function simply defines the left-most digit as the integer to the left of the decimal point. A more complex version would be required to account for greater ranges of values and the other characteristics of the LDE observed in the literature, e.g. smaller jumps at right digits ending in five, or at other psychologically notable price points, etc. Equally, the choice of reference point itself may suffer from the LDE whereby the nearest significant round number might be chosen as the reference point.

${ }^{4}$ For long positions, the investor 'buys' the market to 'open' a position with the expectation that the market will rise and then subsequently 'sells' the market to 'close' their position and realize a profit (if the market has risen) or a loss (if the market has fallen). For short-sell positions the opposite occurs. The investor 'sells' the market to 'open' a position with the expectation that the market will fall and then subsequently 'buys' the market to 'close' their position and realize a profit (if the market fell) or a loss (if the market had risen). Since we are interested in the realization (i.e. closure) of gains/losses we are interested in the over-selling of long positions and over-buying of short positions on round numbers.

5 The relevant ask/bid price for buy/sell trades.

${ }^{6} \mathrm{~A} \$ 50$ per point spread trade on the Dow Jones 30 Index at a price of 17,500 might only require an investor to hold $\$ 2,500$ in capital in the trading account. However, this leverages an $\$ 875,000$ sized position in real terms. 
${ }^{7}$ In spread trading, limit orders are orders in advance to buy or sell an index when the price of the index reaches that point, usually some distance from the current price. Market orders are orders to buy or sell an index at or near to the current price (i.e. best price). 


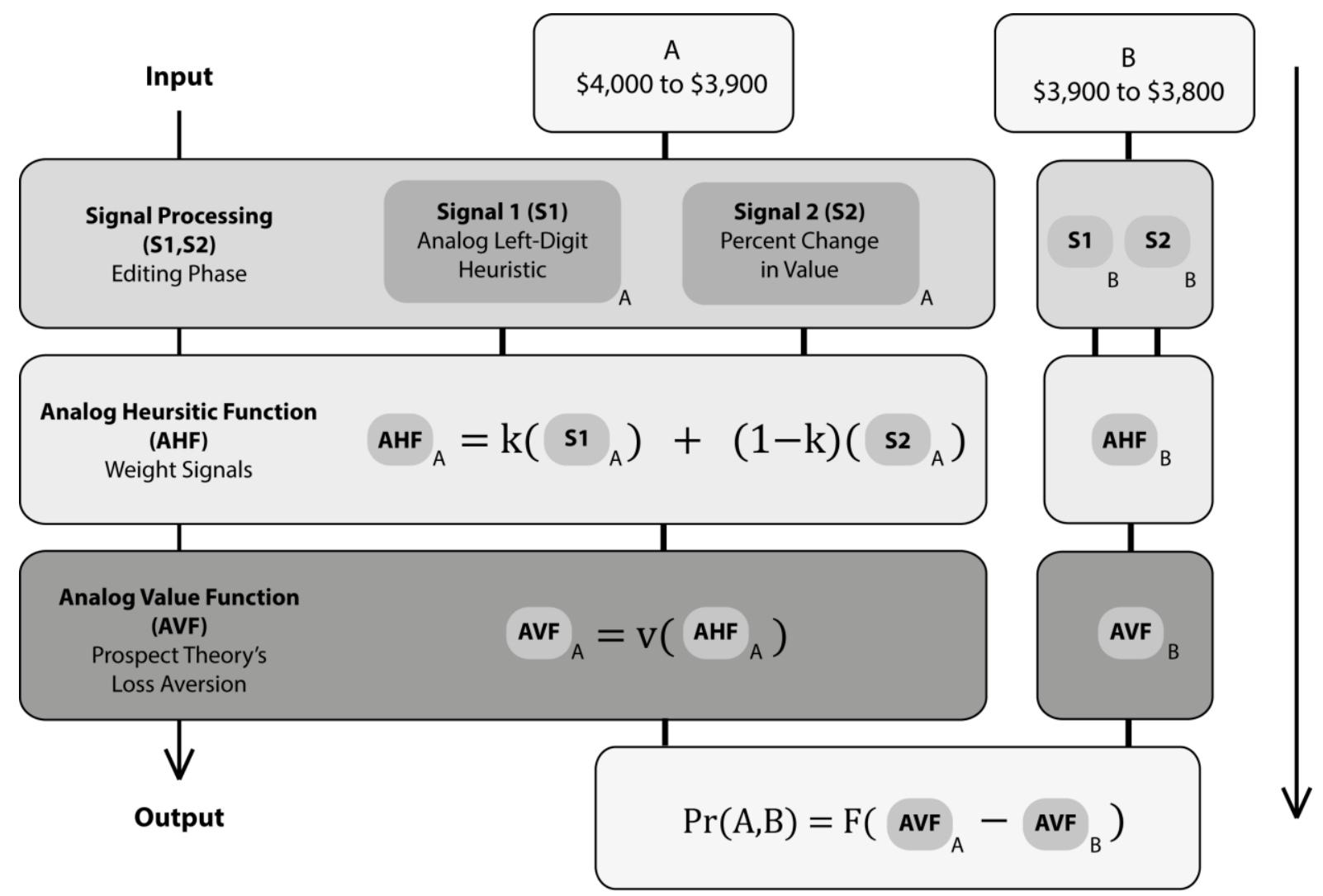

Figure 1. An illustration of the integration of analog heuristic processing with prospect theory to model the LDE bias when comparing two changes in value (A and B). The parameter $k$ determines the extent that changes in left digits are incorporated into the perception of changes in value. Parameter $v$ is prospect theory's value function applied to this heuristic signal to produce an analog value function. Choice probabilities are a function $(F)$ of a comparison of the analog value functions for each alternative. 
(A) $R=5.0, \alpha, \beta=0.5$ and $\gamma=2.25$

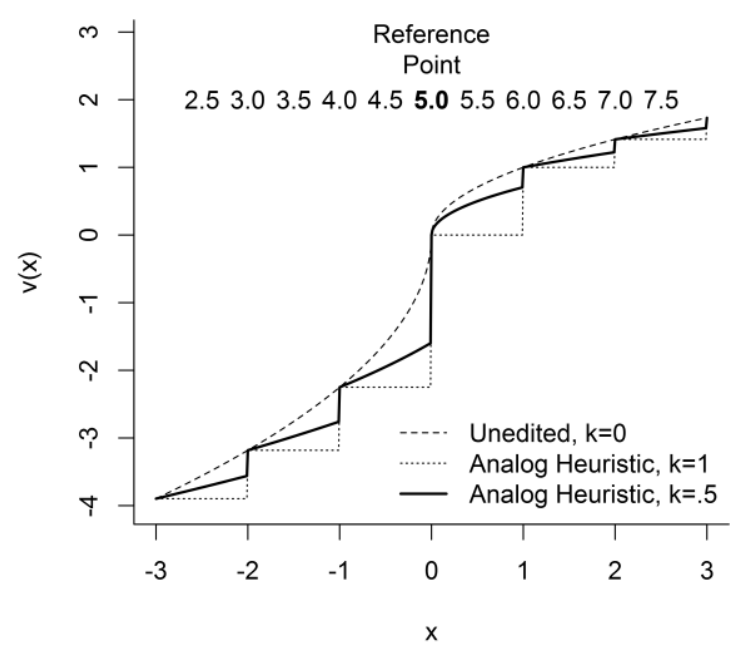

(B) $R=5.5, \alpha, \beta=0.5$ and $\gamma=2.25$

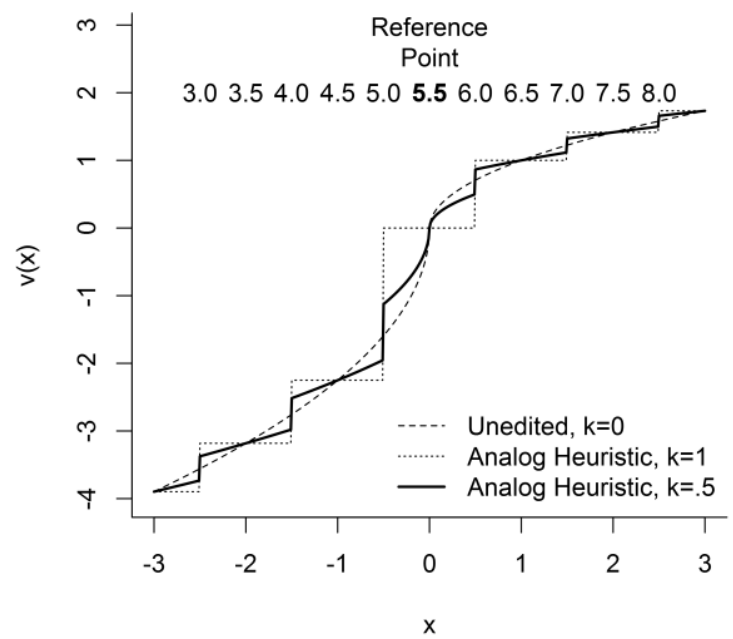

Figure 2. The effect of prospect theory's value function (using typical example values for prospect theory's $\alpha, \beta$ and $\gamma$ parameters) on the change in value (x) from a reference point $\mathrm{R}$ (5.0 in A and 5.5 in B) which is unedited vs. edited via an analog heuristic. The influence of the analog heuristic relative to the actual difference in the value of $\mathrm{x}$ is captured by the value of $\mathrm{k}$. In particular, where $\mathrm{k}=0$, the analog heuristic has no influence (i.e. an unedited, standard prospect theory, function), where $\mathrm{k}=1$, the analog heuristic completely dominates (i.e. only the left-digits are considered) and where $0<\mathrm{k}<1$ there is partial influence of both the true change in value $\mathrm{x}$ and the analog heuristic. 


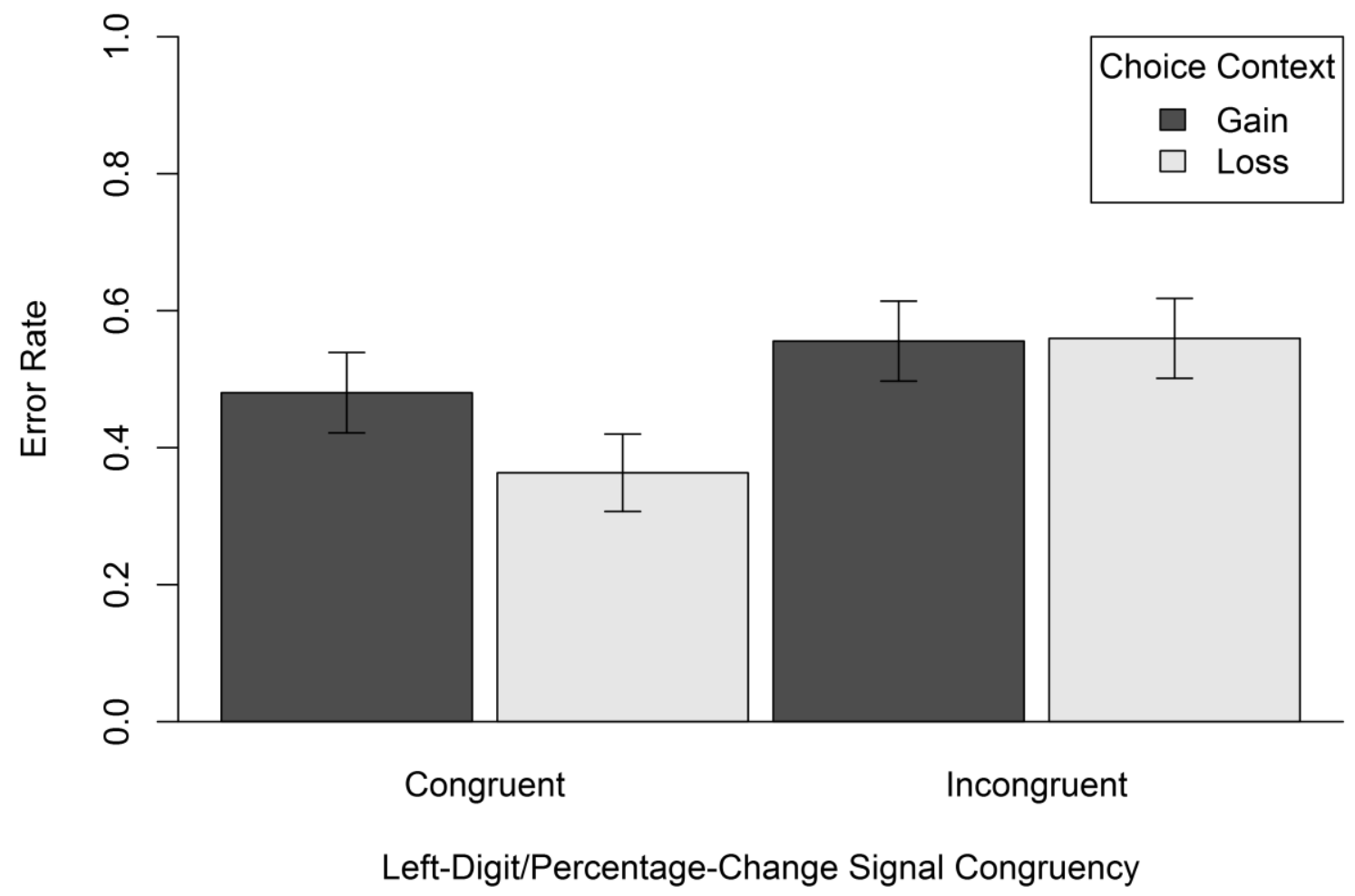

Figure 3. The effect of congruency between the change in left digits and the percentage change in value on the error rate (proportion of selections of the worst performing investment) for gaining and losing investments. 
(A) Positions in Profit

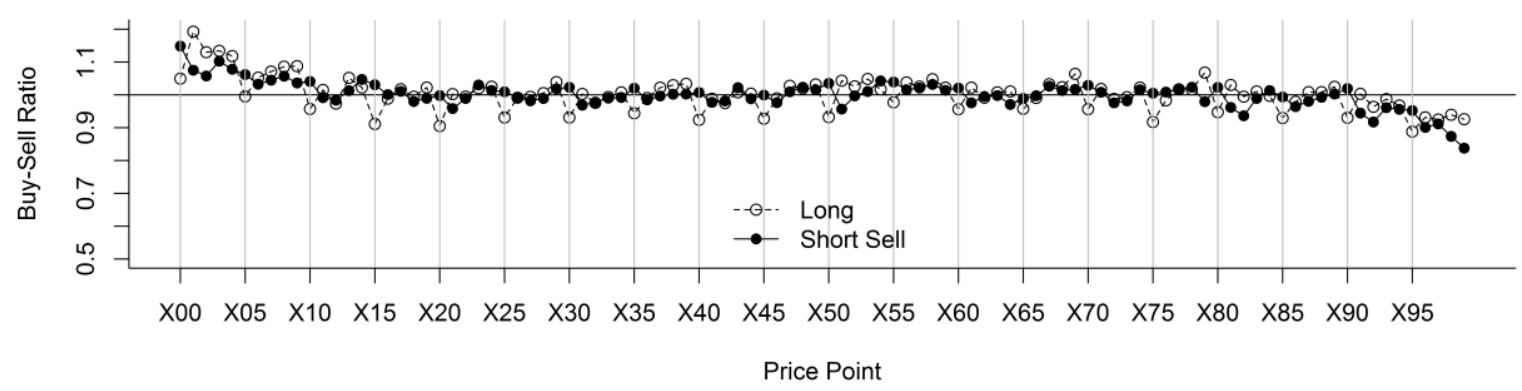

(B) Positions in Loss

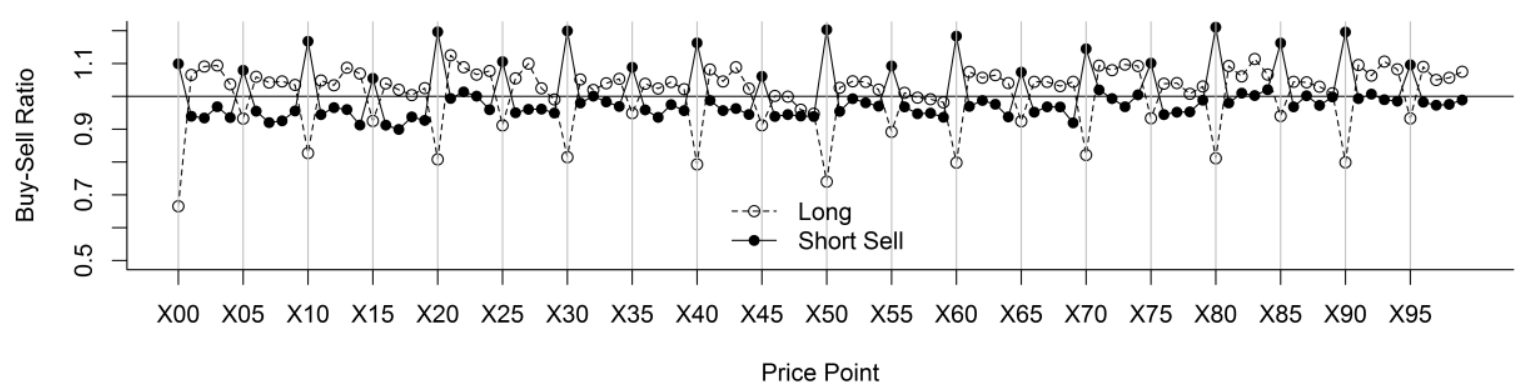

Figure 4. The total number of buys/number of sell trades (Buy-Sell Ratio) for the final two digit price points for long and short positions that were associated with positions in either profit or in loss. Figure based on 7,571,611 trades. 
(A) Positions in Profit

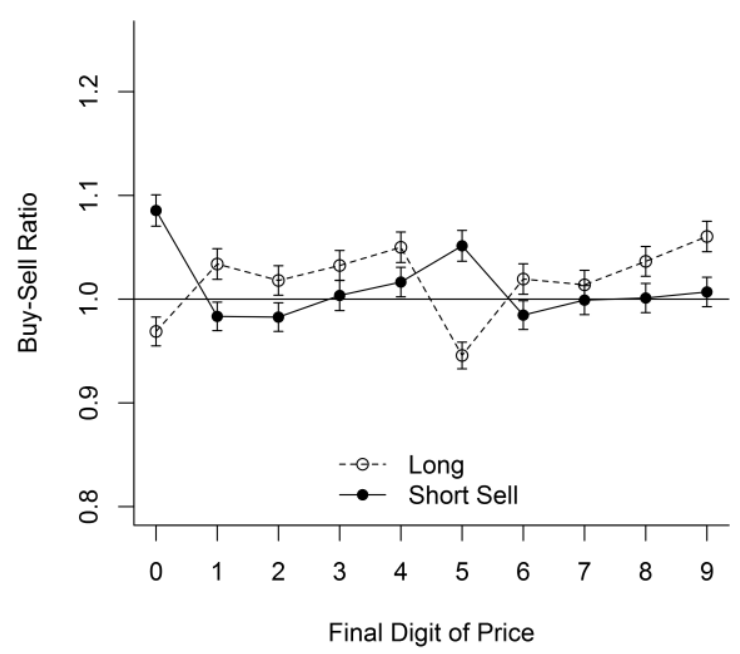

(B) Positions in Loss

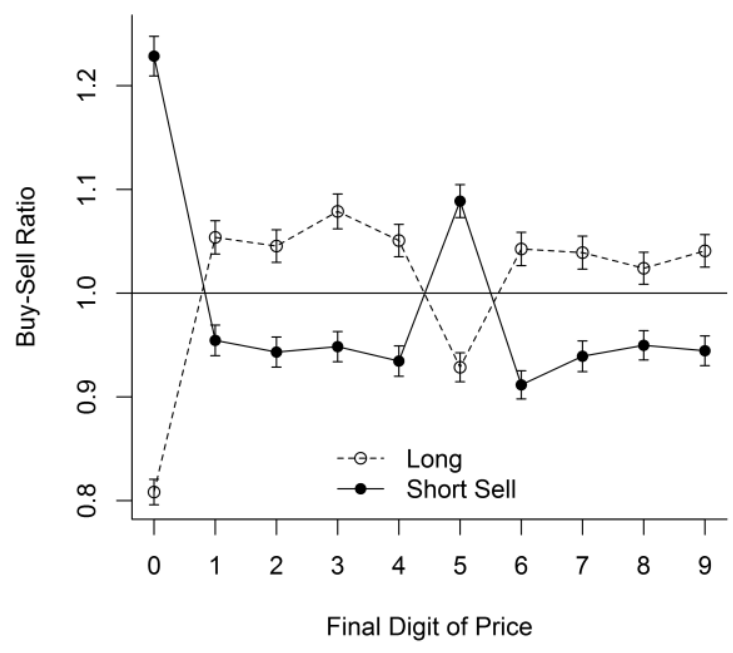

Figure 5. shows the mean (and 95\% confidence interval error bars) of each trader's buys-sell ratios at final digit price points for long and short positions that were either (A) in profit or (B) in loss. 
Table 1

An example of an 'incongruent condition': whereby an asset (A) that gained in value (June to October) by the greatest percentage (A (31\%) vs. B (28\%)), increased by the least number of left digits (A, by 2 digits (29\%) left of the comma separator, 7 to $9 \mathrm{cf}$. B, by 3 digits, (43\%) left of the comma separator, 7 to 10).

\begin{tabular}{|c|c|c|c|c|c|}
\hline Month & IUNE & JULY & $\underline{\text { AUGUST }}$ & $\underline{\text { SEPT }}$ & OCTOBER \\
\hline Asset status & bought & held & held & held & current \\
\hline A & $£ \quad 7,098$ & 7,199 & $£ \quad 8,400$ & 9,101 & $£ \quad 9,302$ \\
\hline B & 7,911 & 8,012 & 9,213 & 9,914 & $£ 10,115$ \\
\hline
\end{tabular}


Table 2

Details of the twelve gain/loss binary choices (assets A vs. B) offered to participants and the percentage of participants who chose assets A and B. For each choice we show the 'value choice', based on the percentage change in value of the asset, and the 'heuristic choice', based on the LDE. The final column indicates choices in which the biased and value-based choices are congruent/incongruent with each other

\begin{tabular}{|c|c|c|c|c|c|c|c|c|c|c|}
\hline Set & $\begin{array}{l}\text { Participant } \\
\text { Choice Rate }\end{array}$ & Stock & $\begin{array}{c}\text { Original } \\
\text { Value }\end{array}$ & $\begin{array}{c}\text { Present } \\
\text { Value }\end{array}$ & $\begin{array}{l}\text { Gain or Loss } \\
\text { Scenario }\end{array}$ & $\begin{array}{c}\text { Change in Value } \\
\text { for Stock, } V\left(x_{i}\right)\end{array}$ & $\begin{array}{c}\text { Value }\left(\Delta \text { Value }_{i}\right) \\
\text { Choice }\end{array}$ & $\begin{array}{c}\text { Change in Left-Digits } \\
\text { for stock, } \mathrm{D}\left(\mathrm{x}_{\mathrm{i}}\right)\end{array}$ & $\begin{array}{c}\text { Heuristic } \\
(\Delta \text { LeftDigitsi) Choice }\end{array}$ & Congruency \\
\hline \multirow{2}{*}{1} & $55.4 \%$ & $\mathrm{~A}$ & 17,911 & 20,115 & \multirow{2}{*}{ Gain } & $12.3 \%$ & \multirow{2}{*}{$\mathrm{B}>\mathrm{A}$} & $17.6 \%$ & \multirow{2}{*}{$\mathrm{A}>\mathrm{B}$} & \multirow{2}{*}{ Incongruent } \\
\hline & $44.6 \%$ & $\mathrm{~B}$ & 17,098 & 19,302 & & $12.9 \%$ & & $11.8 \%$ & & \\
\hline \multirow{2}{*}{2} & $41.9 \%$ & A & 17,010 & 14,806 & \multirow{2}{*}{ Loss } & $-13.0 \%$ & \multirow{2}{*}{$\mathrm{B}>\mathrm{A}$} & $-17.6 \%$ & \multirow{2}{*}{$\mathrm{B}>\mathrm{A}$} & \multirow{2}{*}{ Congruent (B } \\
\hline & $58.1 \%$ & $\mathrm{~B}$ & 17,901 & 15,697 & & $-12.3 \%$ & & $-11.8 \%$ & & \\
\hline \multirow{2}{*}{3} & $50.5 \%$ & A & 18,089 & 20,293 & \multirow{2}{*}{ Gain } & $12.2 \%$ & \multirow{2}{*}{$\mathrm{B}>\mathrm{A}$} & $11.1 \%$ & \multirow{2}{*}{$\mathrm{B}>\mathrm{A}$} & \multirow{2}{*}{ Congruent (B } \\
\hline & $49.5 \%$ & $\mathrm{~B}$ & 17,900 & 20,104 & & $12.3 \%$ & & $17.6 \%$ & & \\
\hline \multirow{2}{*}{4} & $58.7 \%$ & A & 17,908 & 15,704 & \multirow{2}{*}{ Loss } & $-12.3 \%$ & \multirow{2}{*}{$\mathrm{B}>\mathrm{A}$} & $-11.8 \%$ & \multirow{2}{*}{$A>B$} & \multirow{2}{*}{ Incongruent } \\
\hline & $41.3 \%$ & $\mathrm{~B}$ & 18,004 & 15,800 & & $-12.2 \%$ & & $-16.7 \%$ & & \\
\hline \multirow{2}{*}{5} & $60.6 \%$ & A & 97,904 & 100,108 & \multirow{2}{*}{ Gain } & $2.3 \%$ & \multirow{2}{*}{$\mathrm{B}>\mathrm{A}$} & $3.1 \%$ & \multirow{2}{*}{$\mathrm{A}>\mathrm{B}$} & \multirow{2}{*}{ Incongruent } \\
\hline & $39.4 \%$ & $\mathrm{~B}$ & 97,098 & 99,302 & & $2.3 \%$ & & $2.1 \%$ & & \\
\hline \multirow{2}{*}{6} & $66.7 \%$ & A & 102,911 & 100,707 & \multirow{2}{*}{ Loss } & $-2.1 \%$ & \multirow{2}{*}{$\mathrm{A}>\mathrm{B}$} & $-2.0 \%$ & \multirow{2}{*}{$\mathrm{A}>\mathrm{B}$} & \multirow{2}{*}{ Congruent (A) } \\
\hline & $33.3 \%$ & B & 102,001 & 99,797 & & $-2.2 \%$ & & $-2.9 \%$ & & \\
\hline \multirow{2}{*}{7} & $58.1 \%$ & A & 96,902 & 99,106 & $C$ oin & $2.3 \%$ & $A>B$ & $3.1 \%$ & $A>B$ & Congruent (A) \\
\hline & $41.9 \%$ & $\mathrm{~B}$ & 97,030 & 99,234 & Gain & $2.3 \%$ & $A>B$ & $2.1 \%$ & $A>B$ & Congruent $(A)$ \\
\hline 0 & $47.8 \%$ & A & 101,903 & 99,699 & 50 & $-2.2 \%$ & $\mathrm{D}=\mathrm{A}$ & $-2.0 \%$ & 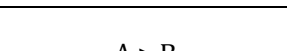 & \\
\hline 8 & $52.2 \%$ & $\mathrm{~B}$ & 102,030 & 99,826 & Loss & $-2.2 \%$ & $\mathrm{~B}>\mathrm{A}$ & $-2.9 \%$ & $\mathrm{~A}>\mathrm{B}$ & Incongruent \\
\hline 9 & $49.5 \%$ & A & 7,098 & 9,302 & Gain & $31.1 \%$ & $A>B$ & $28.6 \%$ & $B>A$ & Inconoruent \\
\hline 9 & $50.5 \%$ & $\mathrm{~B}$ & 7,911 & 10,115 & Gain & $27.9 \%$ & $A>B$ & $42.9 \%$ & $B>A$ & Incongruent \\
\hline 10 & $33.7 \%$ & A & 10,001 & 7,797 & Losc & $-22.0 \%$ & $P>A$ & $-30.0 \%$ & 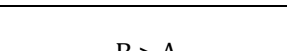 & Conomont (D) \\
\hline 10 & $66.3 \%$ & $\mathrm{~B}$ & 10,911 & 8,707 & Loss & $-20.2 \%$ & $B>A$ & $-20.0 \%$ & $B>A$ & Congruent (B) \\
\hline 11 & $51.6 \%$ & A & 7,098 & 9,302 & $C$ in & $31.1 \%$ & $P>A$ & $28.6 \%$ & $P=A$ & Concruent (D) \\
\hline 11 & $48.4 \%$ & $\mathrm{~B}$ & 6,911 & 9,115 & Grán & $31.9 \%$ & $\mathrm{~B}>\mathrm{A}$ & $50.0 \%$ & $\mathrm{~B}>\mathrm{A}$ & Congruent (B) \\
\hline 12 & $38.7 \%$ & A & 12,001 & 9,797 & Jose & $-18.4 \%$ & $A>B$ & $-25.0 \%$ & $B>A$ & Inconoruent \\
\hline 12 & $61.3 \%$ & B & 11,911 & 9,707 & Loss & $-18.5 \%$ & $\mathrm{~A}>\mathrm{B}$ & $-18.2 \%$ & $B>A$ & Incongruent \\
\hline
\end{tabular}


Table 3

We compare increasingly complex nested models predicting participants choices, with each fixed effect parameter added sequentially to the last model. Loglikelihood ratio (LLR) tests indicate the significance of the main effects of each fixed effect parameter.

\begin{tabular}{lccccc}
\hline & Df & $\Delta$ AIC & LogLik & Chisq. (df) & Sig. \\
\hline$\Delta$ Value & 3 & 0.1 & -771.19 & $1.873(1)$ & .171 \\
LLeftDigits & 4 & -8.0 & -765.23 & $10.05(1)$ & $.002^{* *}$ \\
Loss & 5 & -3.7 & -762.41 & $5.64(1)$ & $.018^{*}$ \\
$\Delta$ LeftDigits $\times$ Loss & 6 & -9.0 & -756.90 & $11.02(1)$ & $.001^{* * *}$ \\
${\text { *** } p<0.001 ;{ }^{* *} p<0.01 ; *}^{*} p<0.05$ & & & &
\end{tabular}


Table 4

The estimated parameters for the traditional value function and the analog value function along with AIC and BIC statistics for two logistic mixed-effects model each using one of these functions to predict participants' choices. Cox test statistics comparing the two non-nested mixed models are shown.

\begin{tabular}{cccc}
\hline Parameter Name & Parameter & $\begin{array}{c}\text { Traditional } \\
\text { Value Function }\end{array}$ & $\begin{array}{c}\text { Analog } \\
\text { Value Function }\end{array}$ \\
\hline Gain power & $\alpha$ & .001 & 0.88 \\
Loss power & $\beta$ & 0.11 & 1.00 \\
Loss aversion & $\lambda$ & 2.25 & 2.11 \\
Analog heuristic weight & $k$ & - & 0.56 \\
\hline & AIC & 1532.3 & 1523.7 \\
& BIC & 1547.3 & 1538.7 \\
& Cox Test & $z=2.73, p=0.098$ & $z=11.42, p<.001$
\end{tabular}

Note: For the Cox Test, fitted values of the current model are regressed as an additional variable added to the alternative model. The test is significant if the fitted values of the current model add significantly to the explanatory value of the alternative model via a log-likelihood ratio test. 
Table 5

Buy-sell ratios for positions currently showing profit or loss for trades with prices ending in five, zero or any other number.

\begin{tabular}{llcll}
\hline & & \multicolumn{3}{c}{ Last Digit of Price } \\
$\begin{array}{l}\text { Position } \\
\text { Outcome }\end{array}$ & $\begin{array}{l}\text { Position } \\
\text { Type }\end{array}$ & Zero & Five & Other \\
\hline Profits & Long & 0.95 & 0.94 & 1.02 \\
& Short & 1.03 & 1.01 & 0.99 \\
\cline { 2 - 5 } Losses & Long & 0.79 & 0.93 & 1.05 \\
& Short & 1.18 & 1.09 & 0.96 \\
\hline
\end{tabular}


Table 6

Coefficients for the fixed effects of the logistic linear mixed effects model estimating the likelihood of long and short positions in profit and in loss at price points ending in five, zero or any other value (reference level)

\begin{tabular}{|c|c|c|c|c|c|}
\hline Fixed Effects & Estimate & Std. Error & $\mathrm{z}$ value & $\operatorname{Pr}(>|z|)$ & \\
\hline (Intercept) & 0.016 & 0.001 & 14.49 & $<.001$ & *** \\
\hline Five & -0.080 & 0.003 & -25.38 & $<.001$ & $* * *$ \\
\hline Zero & -0.069 & 0.003 & -21.9 & $<.001$ & $* * *$ \\
\hline Short & -0.021 & 0.001 & -14.31 & $<.001$ & $* * *$ \\
\hline Loss & 0.031 & 0.002 & 17.51 & $<.001$ & $* * *$ \\
\hline Five $\times$ Short & 0.096 & 0.004 & 21.75 & $<.001$ & $* * *$ \\
\hline Zero $\times$ Short & 0.108 & 0.004 & 24.84 & $<.001$ & $* * *$ \\
\hline Five $\times$ Loss & -0.044 & 0.005 & -8.57 & $<.001$ & $* * *$ \\
\hline Zero $\times$ Loss & -0.219 & 0.005 & -44.1 & $<.001$ & $* * *$ \\
\hline Short $\times$ Loss & -0.061 & 0.002 & -24.99 & $<.001$ & $* * *$ \\
\hline Five $\times$ Short $\times$ Loss & 0.152 & 0.007 & 21.47 & $<.001$ & $* * *$ \\
\hline Zero $\times$ Short $\times$ Loss & 0.378 & 0.007 & 54.84 & $<.001$ & $* * *$ \\
\hline
\end{tabular}




\section{Appendix A: Examples illustrating the effect of analog-based editing of the choice problem}

An investor with high positive expectations standing 'atop a cliff face'

Consider an investor who owns a security and the current price is $\$ 2.00$ (which is considered the reference point by the investor). Let us assume that the investor's subjective probability that the security will rise in price is high (say, $p=.8$ ), and her subjective probability that the market will fall is low $(q=.2)$. Using prospect theory's value function (see eq. 4) and some typical parameter values (i.e. $\alpha, \beta=0.5$, and $\gamma=2.25$ ), for a .01 change in the security's price from the reference point, we can calculate the subjective expected value, $V$, as follows:

$$
\begin{gathered}
V=p v(x)+q v(y), \text { where simply } x=2.01-2.00, y=1.99-2.00 \\
V=0.8(0.01)+0.2(-0.23)=0.04
\end{gathered}
$$

Assuming that the alternative option is to sell the security, with a subjective expected value of zero, prospect theory would predict that the decision-maker should hold or even buy more stock, since $V>0$. However, when we repeat this exercise with analog heuristic-edited inputs for $x$ and $y$, using the heuristic, $h$, from Equation 2 with $k=0.5$ (i.e. now $x=h(2.01,2.00,0.5$ ) and $y=$ $h(1.99,2.00,0.5))$, then

$$
\begin{array}{r}
V=p v(x)+q v(y) \text {, where now } x=0.01, y=-0.51 \\
\quad V=0.8(0.07)+0.2(-1.60)=-0.26
\end{array}
$$

Consequently, having employed an analog-based editing of the choice problem, the investor has a strong preference to sell the stock, as holding is associated with a negative subjective expected value (i.e. $V<0$ ). This results from the large drop in subjective expected value if the price of the stock should fall. Note that this arises despite their strong expectation that the stock will rise in price. 
An investor with moderate positive expectations standing 'at the bottom of a cliff face'

Consider an investor who owns a security and the current price is $\$ 1.99$ (which is considered the reference point by the investor). Let us assume that the investor holds a low subjective probability that the market will rise $(p=.6 ; q=.4)$. Now the subjective expected value, $V$, is as follows:

$$
\begin{gathered}
V=p v(x)+q v(y), \text { where simply } x=2.00-1.99, y=1.98-1.99 \\
V=0.6(0.01)+0.4(-0.23)=-0.09
\end{gathered}
$$

Now the investor does not see any advantage holding onto their position as loss aversion drives them to sell their position $(V<0)$. However, when we repeat this exercise with analog heuristicedited inputs for $x$ and $y$, using the heuristic, $h$, from Equation 2 with $k=0.5$; now $x=h(2.0,1.99$, $0.5)$ and $y=h(1.98,1.99,0.5)$

$$
\begin{aligned}
& V=p v(x)+q v(y), \text { where now } x=0.51, y=-0.01 \\
& V=0.6(0.71)+0.4(-0.16)=0.36
\end{aligned}
$$

Having now employed an analog-based editing of the choice problem the investor has a strong preference to hold the stock $(V>0)$ since there is a large jump in subjective expected value if the stock rises in price. Note that this is despite only a moderate subjective expected probability of the stock rising in price. 